\title{
Vitality Aware Cluster Head Election to Alleviate the Wireless Sensor Network for Long Time
}

\author{
P. Thiruvannamalai Sivasankar \\ Research Scholar \\ Department of Computer Science and Engineering \\ Sathyabama University, India
}

\author{
Dr. M. RamaKrishnan \\ Chairperson and Professor \\ School of Information Technology \\ Madurai Kamaraj University \\ India
}

\begin{abstract}
The Wireless Sensor Networks (WSN) motivated by its unique characters such as it is capable of enduring callous ecological circumstances, and grant better scalability. The wireless sensor network is composed of insignificant sensors and a base station. The battery supplies the energy for the sensors. Hence, the lifetime of the network gets tainted while overworking for transmission. Since the WSN is being utilized for the dangerous purpose, we have to swell the lifespan of the network. The clustering is one of the foremost mechanisms to maximize the network's lifespan. The cluster head assortment plays an imperative role given the fact that clusters head was answerable for the transformation of data between cluster member and the base station. This present article deals with the novel scheme for the cluster head selection entitled as vitality aware cluster head election. In this scheme, the sensor nodes are being clustered into an optimal number. Subsequently, the cluster head is selected by a ballot for each and every group based on its remaining energy. To weigh up the performance of the proposed method, a Network Simulator (NS-2) has been employed.
\end{abstract}

Keywords-Wireless Sensor Networks(WSNs); Residual energy; Clustering; Life span; Sensor

\section{INTRODUCTION}

Tiny sensor nodes and a base station are the principal components of a wireless sensor network. The resource is being bounded by small sensor nodes, capable of sensing the environmental circumstances like pressure, heat, and dampness. The action course of WSN consists of: sensing the environment; sending out the gathered information to the base station and hence processing the information gathered. If the sensor nodes are being disseminated in the sensing meadow, only some of the nodes are in the vicinity of the base station while others may be distant. The energy utilization of the sensor node occurs only during sensing and transmitting it to the base station. Sensor nodes' lifespan is of great significance due to their applications in critical areas.

Numerous schemes were proposed to maximize the lifespan of the network. The clustering is one such scheme exploited to increase the lifespan of the network. The spatially dispersed nodes collectively cluster in a manner that every cluster has a head node (known as the cluster head) which is closer to the base station and its members. Because the energy used for transmission is directly proportional to the distance, lifetime of the network gets increased [1-8].
The paper is structured as follows where Section 1 provides an introduction to the wireless sensor network. Section 2 describes previous studies related to clustering, sensor, and WSN. Section 3 deliberates about the proposed method. Section 4 elaborates the results that obtained through proposed method, and finally the paper concludes with the conclusion and future direction.

\section{RELATED WORK}

Extensive literature survey reveals that numerous schemes were employed for this purpose. For instance, Bai et al. [9] proposed the energy efficient clustering mechanism of LEACH (Low Energy Adaptive Clustering Hierarchy). It is used to shun the contention and diminish the traffic load in the channel. In LEACH, the nodes form the clusters locally, and each and every cluster has one cluster head. The LEACH has two phases namely, (i) a setup stage and (ii) a steady state stage. During the process of a setup stage, nodes of a cluster decide the presence of a cluster head in the cycle. The cycle in WSN points towards the fact that every cluster head gathers information from all their members and relays it to the base station [10]. The choice of cluster head selection is based on the random likelihood of a node to become a cluster head. Every cluster head receives the gathered information sent by sensor nodes of a cluster followed by each cluster head's relay of the collected data to a base station in steady state stages. Election of

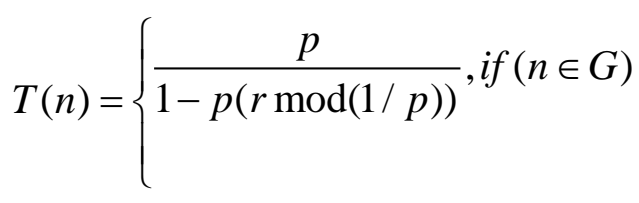

else $\quad T(n)=0$

Liang [11] offered the fuzzy logic based cluster head election method in which the base station picks the cluster head. There are three descriptors used in that method- energy, attentiveness, and centrality. These descriptors help calculate the likelihood for each sensor node to be a cluster head. In the base station, a central control algorithm provides the total information about the network using a selected cluster head.

Maraiya et al. [12] proposed an efficient cluster head selection method where the primary focus was on avoiding reclustering, that minimizes the utilization of energy for 
transmission purpose by attempting to shun overload in the cluster head. In this well-organized cluster head selection scheme, the cluster head is chosen with respect to the remaining energy in the node. Novelty of the present work is that, an associate cluster head is selected, and it becomes a cluster head while the leftover cluster head's energy drops below the energy of the other non-CHs in the cluster. Therefore, this scheme avoids re-clustering mechanism.

Lakshmi and Neelima [13] suggested that the importance of cluster head. The authors emphasized that selection based on the hit sets where the lifespan of the network is augmented by dipping the number of active nodes contributing to the transmission. The following are two chief ways to enhance the extent of the network's lifetime: optimizing the communication and reducing the energy usage. An efficient way to optimize the communication is to elect the cluster head efficiently. The hit set identifies the active nodes, and one of the same becomes the cluster head by its node degree. Similarly, Kumar et al. [14] and Kumar and Prabha [15] proposed that the location based clustering mechanism. The sensor that is near the base station is being chosen as cluster head.

Findings of these studies indicated that the clustering is the efficient way to enhance the network's lifespan, and the cluster head selection plays a crucial role. In this paper, the vitality aware cluster head selection mechanism was used to pick out an appropriate cluster head to reduce selection overhead of the cluster head. The choice of the cluster head is based on its residual energy and the probability of being a cluster head. The performance of the anticipated method was being proved by the analysis of the simulation results obtained from NS2 [16].

\section{PROPOSED WORK}

The wireless sensor network finds several usages which include disaster recovery, military application, and fire detection system and temperature monitoring system. Tiny sensors and a base station couple together to constitute a wireless network. The sensor runs on the battery power. Therefore, the lifetime of the network gets degraded while overloading the system.

As the WSN has been used widely in the critical application, there is a need to increase the network's lifetime. The clustering is the primary technique for enhancing the longevity of the network. The choice of a cluster head plays a vital part as every cluster head is accountable on the statement of data sensed by its members to its base station. Figure.1 illustrates clearly the method adopted in the present study. In our proposed method, all sensor nodes present in the network group together as a cluster into a best possible number, followed by the cluster head election for each cluster.

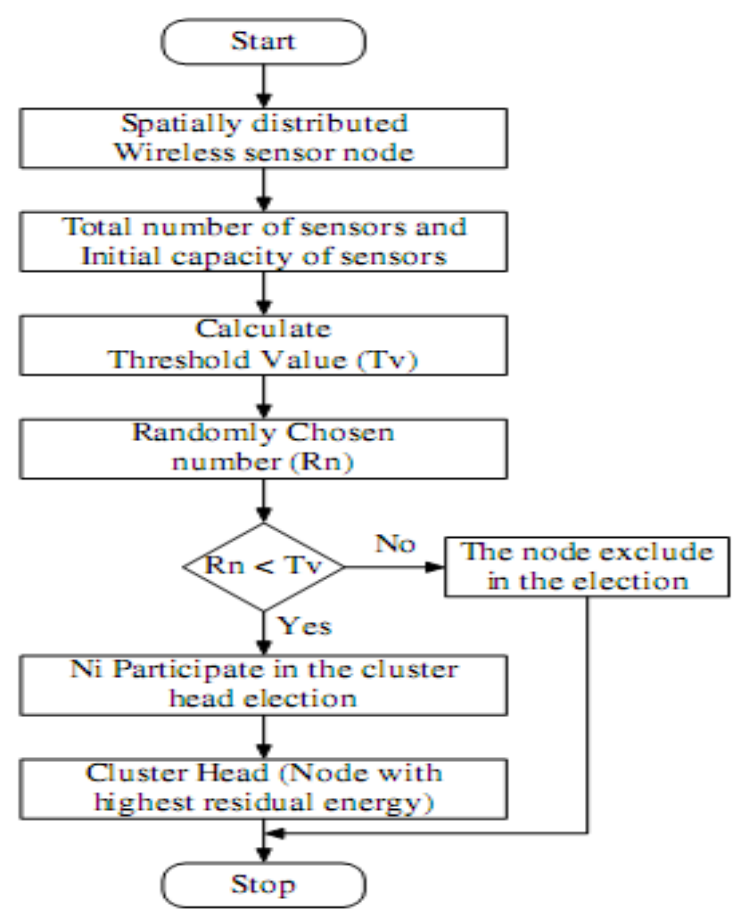

Fig. 1. Block diagram of Vitality aware cluster head election method

To frame the network, the optimal number of clusters required is guesstimated by using the formula given below

$$
N_{\text {Cluster }}=\frac{\text { Total number of sensors }}{\text { Initial capability of a sensor }}
$$

The present research work considers the distribution of sensors in a circular region with the base station at the center.

A. Cluster formation

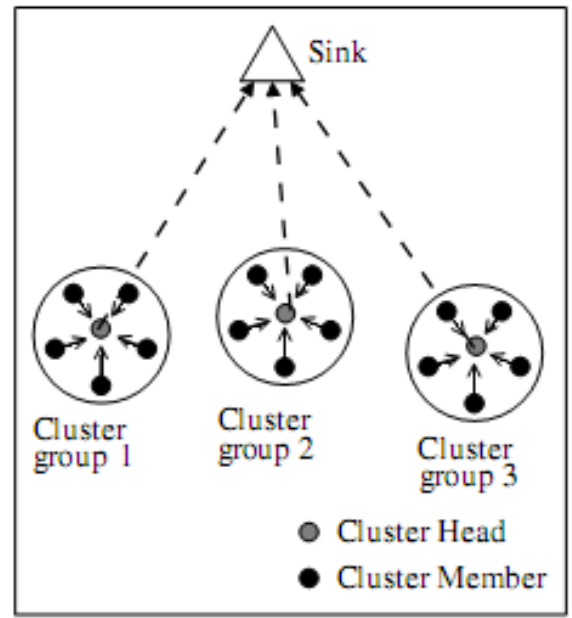

Fig. 2. Cluster Head to Base Station 
The nodes are being grouped into an appropriate number of clusters with regard to its base station. The circular region is divided equally by using the following formula:

$$
\text { Degree }_{\text {Partition }}=360 /_{N_{\text {Cluster }}}
$$

The circular region is being partitioned into equal spaces with the angle between each region Degree $_{\text {Partition. The }}$ optimal number of clusters is formed based on the geographical area.

1) Cluster Head Selection Algorithm

Step1: Cluster head election

for $(j \leftarrow 0$ to $\mathrm{k}$ ) do $j \leftarrow j+1$

Where $\mathrm{k}$ - means number of cluster groups

\{

for ( $i \leftarrow 0$ to $n$ ) do $i \leftarrow i+1$

Where n- means the number of cluster members per cluster group

\{

Finding the cluster head in the clusters was based on the high initial energy through the comparisons between the clusters of the initial energy

$$
\begin{aligned}
& \text { if }\left(C_{M}[0]<C_{M}[i]\right) \\
& \{ \\
& C_{M}[0]=C_{M}[i] ; \\
& \} \\
& C_{H}[j]=C_{M}[0] ;
\end{aligned}
$$

// Selected highest initial energy as a

\section{Cluster Head}

\}

Step 2: Cluster head formation

for $(j \leftarrow 0$ to $\mathrm{m}$ ) do $j \leftarrow j+1$

Where $\mathrm{m}$ - Number of Cluster heads

\section{\{}

for ( $i \leftarrow 0$ to $n$ ) do $i \leftarrow i+1$

Where $\mathrm{n}$ - Number of cluster members

\{

$$
C_{H}=\text { ClusterHead }
$$

$C_{M}=$ ClusterMember

$$
C_{H}[j] \leftarrow C_{M}[i]
$$

Information sent from Cluster member to Cluster Head

$$
\text { \} }
$$

\}

Step 3: Energy calculation at the time of Information sent from $C_{M} \rightarrow C_{H}$
$C_{M}[A E]=C_{M}[I E]-C_{M}[E U]$

Where AE-Available energy, IE-Initial energy, and EU Energy used

Step3: Energy calculation at the time of Received information sent from $\mathrm{C}_{\mathrm{H}}$ to Base Station

for $(j \leftarrow 0$ to $\mathrm{m})$ do $j \leftarrow j+1$

\{

$B S \leftarrow C_{H}[j]$

$C_{H}[A E]=C_{H}[I E]-C_{H}[E U]$

Where BS-Base Station AE-Available energy, IE-Initial energy and EU -Energy used

\}

Step 4: Total Energy used

for $(i \leftarrow 0$ to $\mathrm{n}$ ) do $i \leftarrow i+1$

begin

$C_{M}\left[T_{E U}\right]=T_{C M}[E U]-T_{C H}[E U]$

Where $T_{E U}$-Total Energy Used

/ / $T_{C H}$-Total cluster member

/ / $T_{C H}$-Total cluster heads

end.

2) Distance Estimation from the cluster member to cluster head

Distance matrix (DM) form is given as follows

$$
D M=\left(\begin{array}{ccc}
d_{C_{1}, a_{1}} & \cdots & d_{C H_{n}, a_{n}} \\
\vdots & & \vdots \\
d_{C H_{m}, a_{m}} & \cdots & d_{C H_{m}, a_{n}}
\end{array}\right)
$$

\section{Distance Estimation Algorithm}

Step1: Initialize the cluster member $\mathrm{n}$ in each cluster within a circular region.

cluster $\mathrm{k}=1$

Step2: Select the cluster head based on the highest residual energy within cluster members in each cluster group. Assume the number of cluster head is $\mathrm{m}$ in a circular region

Step 3: The distance of a cluster member to its base station is determined through its cluster heads

$$
\begin{aligned}
& \text { for }(i=0 ; i<m ; i++) \\
& \quad \text { Where } m-\text { means the number of cluster heads } \\
& \{ \\
& \mathrm{K}:=1 ; \\
& \{ \\
& \operatorname{for}(j=0 ; j \leq n ; j++)
\end{aligned}
$$$$
\{
$$$$
\{
$$ 
// Where $\mathrm{n}$ is the number of members in each cluster \{

$d_{1}(0,0)=0$

$d_{i}(j, B S)=d_{i j}(C H[i], j)+d_{i j}(B S, C H[j])$;

\}

$\mathrm{K}=\mathrm{K}+1$;

\}

\section{B. Energy Consumption Model}

Heinzelman et al. [17, 18] applied the Energy Consumption Model as shown in the Figure 3.

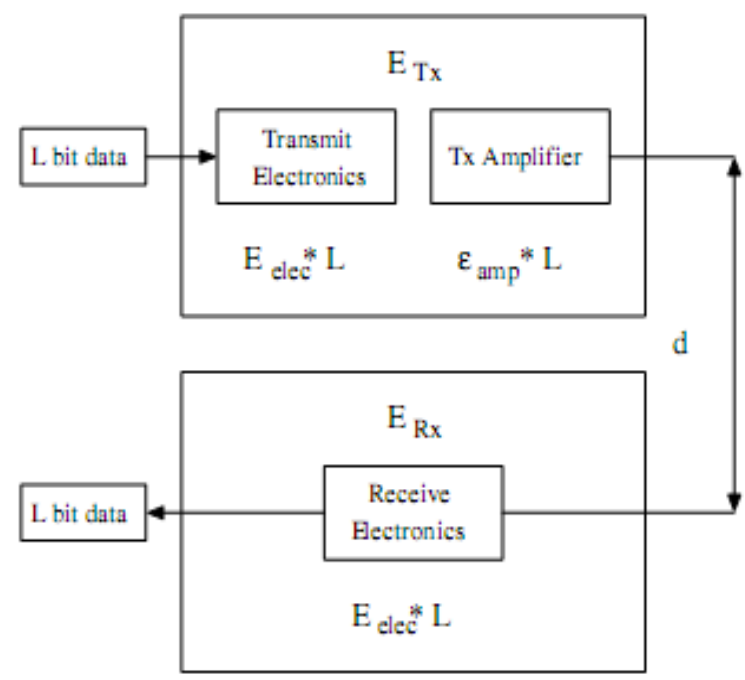

Fig. 3. Energy Consumption Model

The Energy consumption Model in Wireless Sensor Network consists of three parts. The parts are as follows:

1) The sending l-bit message at distance $d$ requires energy.

$$
E_{T X}(l, d)=\left\{\begin{array}{c}
l * E_{\text {ele }}+l^{*} \varepsilon_{\mathrm{fs}} * \mathrm{~d}^{2}, d \leq d_{0} \\
l * \text { Eele }+l * \varepsilon_{\mathrm{mp}} * \mathrm{~d}^{4}, d \geq \mathrm{d}_{0}
\end{array}\right.
$$

Where $E_{\text {ele }}$ is circuit fatigue of sender and receiver.

$d_{0}$ is the critical distance of multipath fading model and free space model

$\varepsilon_{\mathrm{fs}}$ is the amplifier coefficient of free space model

$\varepsilon_{\text {mp }}$ is the multipath fading model

2) Receiving l-bit message at a distance $d$, requires energy

$$
E_{R X}(l)=l * E_{e l e}
$$

3) Data aggregation of energy is

$$
E_{D A}=l * E_{d a}
$$

\section{Vitality aware cluster head election}

After grouping the sensors into clusters, each and every node in the cluster was selected at a random number between zero and one. In case, the random number is lower than the threshold value (n), each node participates in the process of election otherwise it excludes itself from the election process. The threshold value is ascertained by using the formula given below

$$
\operatorname{Threshold}(n)= \begin{cases}p\left(E_{\text {Current }} / E_{\text {Average }}\right) & \text { if } n \in C i \\ 0 & \text { Otherwise }\end{cases}
$$

Where $\mathrm{p}$ is the node as $\mathrm{n}$ is the probability of being cluster head. $E_{\text {Current }}$ Denotes the current energy of a node n. $E_{\text {Average }}$ Indicates that the average energy of nodes in the cluster $\mathrm{Ci}$.

If a cluster consists of several nodes, a particular node is chosen in a manner that, that node becomes a cluster head. Otherwise the node that is very close to the base station with high residual energy is elected as a cluster head.

For each and every round, the cluster head is reelected. The elected cluster head, gathers the sensed information from all of its members in the cluster. Then the cluster head transmits the collected information to the base station. As the cluster head is selected based on its energy level distance to the base station and its probability of being the cluster head, the proposed method increases the existence of the network and also reduces the clustering overhead which takes place in the wireless sensor network.

\section{SiMULATION RESULTS}

The efficiency of the adopted method was analyzed by using a discrete event time driven Network Simulator (NS2). Each and every function of the network is called the event. The programmer explicitly gives the time at which the event should occur. This simulator displays the result in an animated format using the Network animator. The network simulator traces all the network events dynamically to a trace file by which the graph plotted. The following graphs obtained from the simulation of the vitality aware cluster head election scheme (VACS) are used to grade the performance of the network. The parameters like packet delivery rate, packet loss rate, and residual energy has been plotted.

The packet delivery rate was calculated by using the following formula,

$$
=\frac{\text { No. of packets sent }- \text { No. of packets dropped }}{\text { Time }}
$$

Figure 4 shows the graph of the packet delivery rate. 


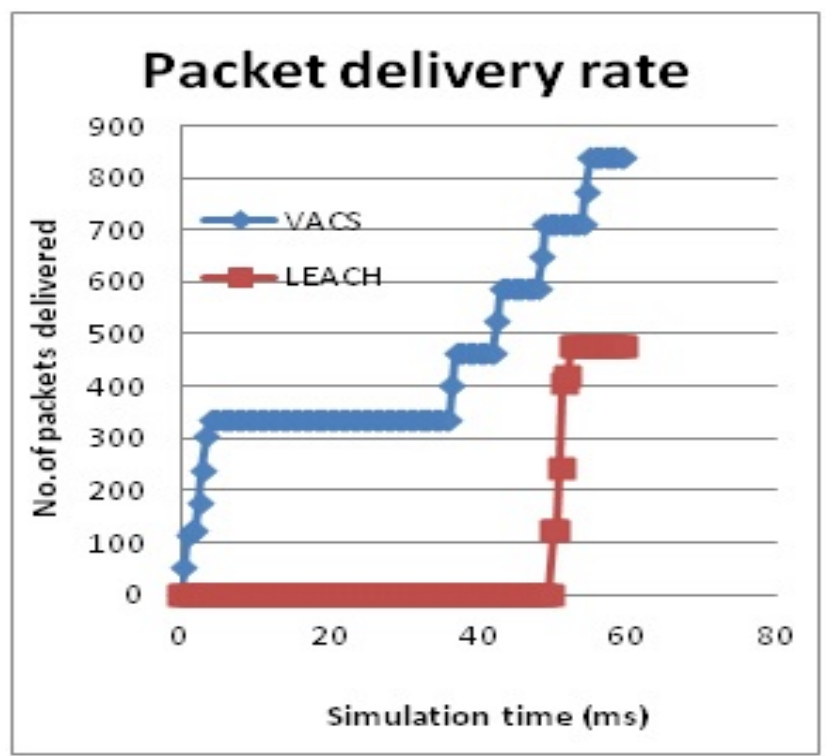

Fig. 4. Packet delivery rate analysis

The proposed system is evaluated by comparing its performance with the standard clustering scheme LEACH. The graph shows that the packet delivery rate of the proposed method is $35 \%$ higher than LEACH clustering scheme. Therefore, the throughput of the system is increased.

The packet loss rate of the system was calculated using the formula

\section{Packet loss rate}

$=\frac{\text { No. of packets sent }- \text { No. of packets received }}{\text { Time }}$

The packet loss occurs due to the destination being out of range and the packet arrival rate being higher than the queue size.

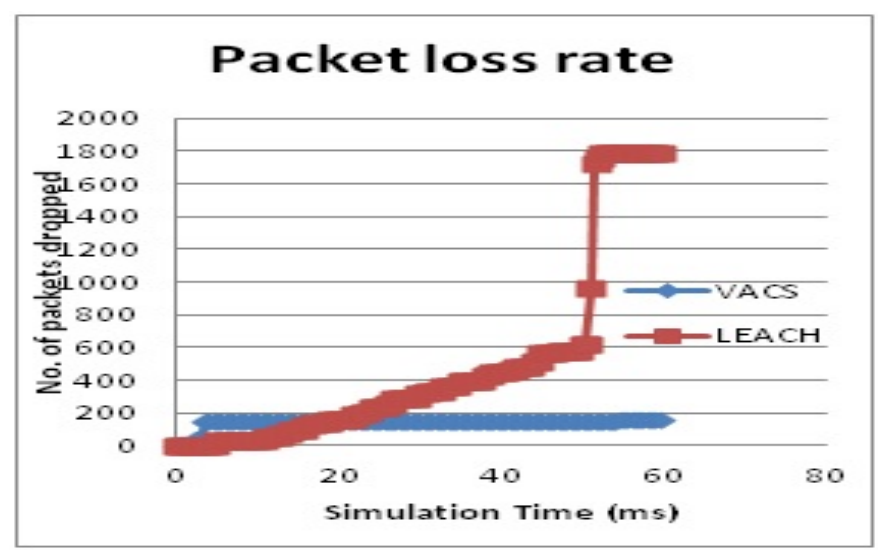

Fig. 5. Packet loss ratio analysis

The graph of the packet loss rate in Figure 5 shows that the proposed system supersedes the standard clustering mechanism LEACH. The residual energy was used to calculate the lifetime of the network. The residual energy was the remaining energy in a node after some events occur. The following formula was used to calculate the Residual energy:
Where,

$$
\text { Residual energy }=E_{\text {initial }}-E_{\text {Tranmission }}
$$

$E_{\text {initial }}$ denotes the initial energy

$E_{\text {Tranmission }}$ denotes the energy used for transmission

The mean value of residual energy in every node of a network was calculated for each $0.5 \mathrm{~ms}$ until the simulation ends.

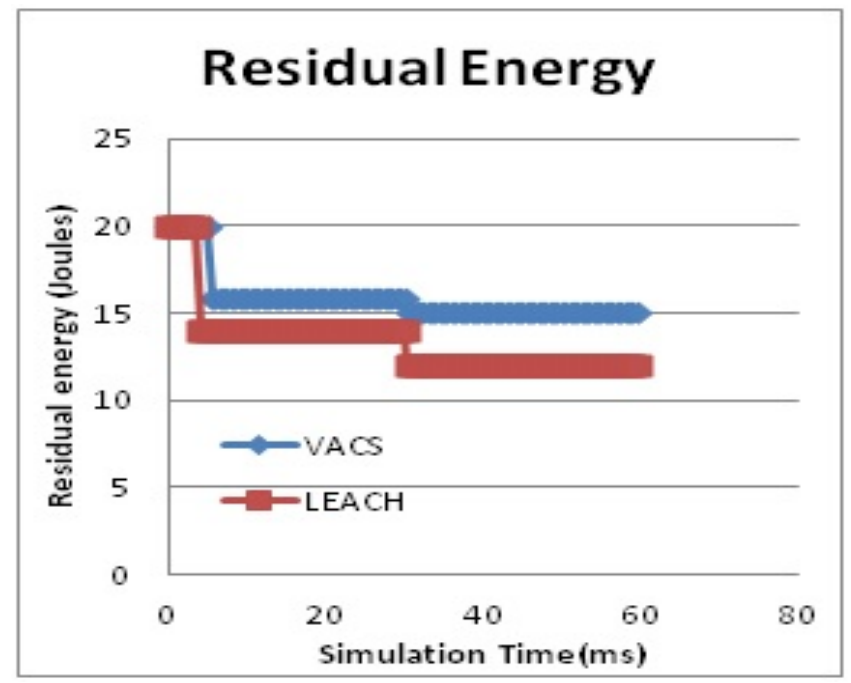

Fig. 6. Residual energy of the sensors after one round

The calculated energy has plotted as shown in Figure 6 and compared to the LEACH clustering mechanism. The findings showed that the proposed scheme outperforms than the LEACH.

\section{CONCLUSION}

This paper illustrated the expectation of a novel cluster based head election method. The method considered the likelihood value of a node being the cluster head to formulate the sensor node and to contribute to the election process. Remaining energy and the remoteness of the base station are used during the process of election head, which thereby prevents the participation of sensor nodes. Therefore, this method shuns the disqualified node to participate in the election process and subsequently, increases the lifespan of the network and reduces the cluster head selection overhead.

\section{REFERENCES}

[1] I.F. Akyildiz, Weilian Su, Y. Sankarasubramaniam, and E. Cayirci, "A survey on sensor networks,” IEEE Commun. Mag., vol. 40, pp. 102-114 2002.

[2] I.F. Akyildiz, W. Su, Y. Sankarasubramaniam,and E. Cayirci, "Wireless Sensor Networks: A Survey,” Computer Networks, 38(4): 393-422, 2002.

[3] .K. Akkaya and M. Younis, “A survey on routing protocols for wireless sensor networks,” Ad Hoc Networks, vol. 3, no. 3, pp. 325-349, 2005.

[4] C.Y. Chong, S.P. Kumar, B.A. Hamilton "Sensor Networks: Evolution, Opportunities, and Challenges,” Proceedings of IEEE, 91(8):1247-1256, 2003.

[5] B.K. Debroy, M.S. Sadi and M. A1 Imran, "An Efficient Approach to Select Cluster Head in Wireless Sensor Networks,” J. Commun., vol. 6, pp. 529-539, 2011. 
[6] A.A. Abbasi and M. Younis, "A survey on clustering algorithms for wireless sensor networks,” Comput. Commun., 30, pp. 2826-2841, 2007.

[7] J.-Y. Chang and P.-H. Ju, "An efficient cluster-based power saving scheme for wireless sensor networks," EURASIP J. Wirel. Commun. Netw., vo. 2012, pp. 172, 2012.

[8] B. Singh and D. Lobiyal, "A novel energy-aware cluster head selection based on particle swarm optimization for wireless sensor networks," Human-centric Comput. Inf. Sci., vol. 2, pp. 13, 2012.

[9] F.e. Bai, H.h. Mou and J. Sun, "Power-efficient zoning clustering algorithm for wireless sensor networks," In: International Conference on Information Engineering and Computer Science (ICIECS 2009) pp. 1-4, 2009.

[10] H. Zhang, S. Zhang and W. Bu, "A Clustering Routing Protocol for Energy Balance of Wireless Sensor Network based on Simulated Annealing and Genetic Algorithm,” Int. J. Hybrid Inf. Technol., vol. 7, pp. 71-82, 2014.

[11] Q. Liang, "Cluster head election for mobile ad hoc wireless network," In: Proc. 14th IEEE International Symposium on Personal, Indoor and Mobile Radio Communications, (PIMRC), pp. 1623 -16, 2003.
[12] K. Maraiya, K. Kant and N. Gupta, "Efficient Cluster Head Selection Scheme for Data Aggregation in Wireless Sensor Network," Int. J. Comput. Appl. (0975 - 8887). vol. 23, pp. 10-18, 2011.

[13] B.J. Lakshmi and M. Neelima, "Maximising Wireless Sensor Network lifetime through cluster head selection using Hit sets," IJCSI Int. J. Comput. Sci., issue 9, pp. 328-331, 2012.

[14] A. Kumar, N. Chand and V. Kumar, "Location Based Clustering in Wireless Sensor Networks,” World Acad. Sci. Eng. Technol., vol. 5, pp. 1313-1320, 2011.

[15] N. Kumar and V.S. Prabha, "Comparative analysis of energy-efficient cluster-based routing protocols for wireless sensor networks," Sensors and Transducers, vol. 142, pp. 23-32, 2012.

[16] J. Ferdous, M.J. Ferdous and T. Dey, "A Comprehensive Analysis of CBCDACP in Wireless Sensor Networks,” J. Commun., vol. 5, pp. 627636, 2010.

[17] W.B. Heinzelman, A.P. Chandrakasan and H. Balakrishnan, "An application-specific protocol architecture for wireless microsensor networks," IEEE Trans. Wirel. Commun., vol. 1, pp. 660-670, 2002.

[18] W.R. Heinzelman, A. Chandrakasan and H. Balakrishnan, "Energyefficient communication protocol for wireless microsensor networks," In: Proceedings of the 33rd Annual Hawaii International Conference on System Sciences,vol 2, pp. 10, IEEE Comput. Soc., 2000. 